\title{
Halitosis, diagnosis and management in daily practice: Dentist stance
}

\author{
Dr. Druva Kumar.Gani ${ }^{1}$, Dr.Ram Babu Dudala ${ }^{2}$ Dr. Ramesh Babu Mutthineni ${ }^{3}$, \\ Dr. Chandra Mohan Pabolu ${ }^{4}$ \\ 1(Dept.of Periodontics/Gandhi Dental College/Orissa/India) \\ 2(Dept.of Periodontics/ Gurunanak Institute of Dental Sciences \& Research/WestBengal/India) \\ 3,4(Dept.of Periodontics/Mamata Dental College/Khammam/India)
}

\begin{abstract}
Halitosis or bad breath or breath malodor is the problem faced by many and can limit their potential to even interact with the society in a positive manner. It has important socioeconomic consequences and can reveal important diseases. It is very subjective in that for many this malady is" A perception rather than a real thing, everybodys breath smells to certain extent" However real halitosis is an unpleasant condition which creates huge embarrassment with potentially grave consequences. Most of the individual suffering from halitosis seek help from general practioners initially, not the dentist.
\end{abstract}

Key Words: Halitosis, Volatile sulphur compounds

\section{Introduction}

Halitosis is an unpleasant odour that emanates from the oral cavity and can be serious enough to cause personal embarrassment. In the literature, bad breath has been typically referred to as oral malodor or halitosis. The word "halitosis" comes from the Latin "halitus", which means "breath" and the Greek suffix "osis", to specify a condition or a process. According to more recent published studies, bad breath is anything but abnormal. The problem of halitosis has been reported for many years. References were found in Papyrus manuscripts dating back to $1550 \mathrm{BC}$. Hippocrates mentioned that any girl should have a pleasant breath, making sure always to wash her mouth with wine, anise and dill seeds ${ }^{[1]}$. Almost every individual awakes with bad breath each morning. One experiences oral malodor after any prolonged period of decreased salivary flow ${ }^{[2]}$ as in fasting or sleeping ${ }^{[3]}$ therefore any drugs which diminish salivation may have the same effect. While ordinary healthy breath has been described as smelling like "blooming chestnuts" variety of ways depending on the cause. Mouthwash is a generally well accepted and popular way of dealing with oral malodour. The reported prevalence of these causes is manifested in the widespread use of products designed to make us temporarily socially acceptable. Millions of people indulge in self-treatment. The species of microorganisms implicated most often in oral odor are Bacteroides, Fusobacterium, and Klebsiella, although other species can also be involved. These anaerobic organisms multiply in the mouth where the lack of oxygen supports their survival. They thrive on dental plaque, the sticky substance which gathers on teeth, in mouth areas such as the gingival crevice which is the space between the surface of a tooth and the overlapping gum, and the tongue. They also multiply in any saliva that becomes trapped in periodontal defects, or in any area outside of the mainstream of salivary flow. However, this review summarizes the classification, etiology, diagnosis, treatment and hints are given for dentists how to mange in daily practice. Although this classification "fig-1" has not been universally accepted by all experts, there is general agreement that halitosis can be categorized as genuine halitosis, pseudo-halitosis and halitophobia ${ }^{[5]}$. However genuine halitosis has been further subclassified as physiologic halitosis in which there is no readily evident disease or pathological condition, or pathologic halitosis which occurs as an effect of an infective process of the oral tissues. Pseudo-halitosis is a condition in which there is absence of halitosis but the patient believes that they have oral malodour. Halitophobia can occur when there is no physical or social evidence to suggest that halitosis is present and which can persist after treatment for either genuine halitosis or as pseudo-halitosis.

\section{Aetiology And Prevalence}

Bad breath or halitosis is an offensive odor exhaled by the mouth, nasal cavities or facial and pharyngeal sinuses. The reliability of epidemiological data has been questioned, but the prevalence of halitosis has been reported to be as high as 50\%. In a reported study $24 \%$ of patients complained of oral malodour in Japan ${ }^{[6]}$ although in France it has been reported that between 50\%and $60 \%{ }^{[7]}$. It is now widely accepted that halitosis originates from the oral cavity ${ }^{[8,9]}$. Accumulation of bacteria and food residues at the posterior part and in the furrows of the tongue ${ }^{9}$ has considered the major cause ${ }^{[11]}$. Inter-dental plaque and gingivitis may also play a fundamental role, and although periodontal pockets may produce putrid odours, which are still unclear 
${ }^{[12]}$. Halitosis causing bacteria are the primary sources of volatile sulphur compounds, and the chief components are methyl mercaptans and hydrogen sulphide ${ }^{[13]}$. Volatile sulphur compounds and other additional odours such as putrescine, indole, skatole, and cadaverine ${ }^{[14]}$ are generated during the bacterial metabolic degradation of food debris, saliva proteins, desquamated cells, dental plaque and microbial putrefaction ${ }^{[15]}$. The periodontal pocket also provides an ideal environment for volatile sulphur compounds production. The intensity of clinical halitosis has been revealed to be considerably associated with the amount of intraoral volatile sulphur compounds level and directly with periodontal health status ${ }^{[16,17,18]}$.

\section{Diagnosis}

The clinical assessment of oral malodour has been usually subjective, and should be based on smelling the exhaled air of the mouth and nose and comparing the two which also popular as organoleptic assessment. Odour detectable from the mouth but not from the nose, which may likely to be of oral or pharyngeal origin. Odour from the nose alone is likely to be coming from the nose or sinuses ${ }^{[19]}$ rare instances when the odour from the nose and mouth are of similar intensity, moreover a systemic cause may also may likely possible for the malodour. Assessment of the quality of the odour relies on the use of qualified clinicaians ${ }^{[20,21]}$. Objective measurement of the breath components is rarely used in regular clinical practice, as it is time consuming and cost effective. Volatile sulphur compounds can be measured by using a portable sulphide monitor, but as oral malodour may comprise agents other than volatile sulphur compounds this may provide an inaccurate assessment of the source and intensity of oral malodour. However, the potential method of determining the components of halitosis is gas chromatography, but this is not of practical clinical application. Correspondingly, dark field microscopy, the detection of trypsin-like activities of bacteria the benzoylarginine-naphthylamide test, and real time quantitative polymerase chain reaction detection of possible causative oral microbes falls outside the routine clinical assessment of halitosiis. Organoleptic measurement by trained clinicians is considered to be the reference, and the most reliable way of evaluating malodour, although this has been contested by studies showing that measurements with the halimeter appear to be more reproducible ${ }^{[22]}$. Measurement of volatile sulphur compounds levels can be carried out by a variety of methods. Organoleptic which are considered subjective by some clinicians but are the ones of most relevance to patients ${ }^{[23]}$, and the more complex gas chromatography techniques ${ }^{[24]}$. Furthermore, portable computerized volatile sulphur compound monitors or halimeters are now available, which are easy to use and relatively economical. ${ }^{[25]}$ However, the limitations of halimeters are that they have low sensitivity for one of the other sources of malodour, methyl mercaptan and nevertheless a high sensitivity for hydrogen sulphide.

\section{Management}

The successful management of halitosis appears to be pivot on the reduction of volatile sulphur compounds levels, and some other foul volatiles, hence the majority focus on chemical and mechanical options. Mechanical interventions like brushing, flossing and tongue scraping are intend to reduce the reasonable amount of bacteria which produces of volatile sulphur compounds, persistent food matter and cellular debris from the gingiva and tongue. In a recent systematic review explained the effectiveness of tongue scraping for treating halitosis. ${ }^{[26]}$ However, the authors concluded that the mechanical tongue cleaning with tongue scrapers appeared to have very limited and short come benefits for the patients in controlling halitosis. However, the limitations of mechanical methods like tongue scarping have been effective if removal of volatile sulphur compounds producing bacteria from all oral biological sites. The option of mouthrinses may be more effective in reaching the less accessible parts of the oral cavity. Moreover, their greater social acceptance and ease of use has led to the development of a large number and range of over the available mouthrinses ${ }^{[27]}$. A number of mouthrinses contain antibacterial agents in addition to flavoring agents. Components which neutralize can further be divided into those that affect the bacteria directly or the chemical compounds they produce and include chlorhexidine, triclosan, alcohol, chlorine dioxide, phenol, and metal ions like zinc ${ }^{[28,29]}$. Furthermore, some of the odour masking agents consists of essential oils, which can also provide a competing and purely temporary smell that is capable of disguising the unfavorable malodour.

\section{Discussion}

Halitosis is widespread and may consider affecting one quarter of the population around the world. Dental practitioners should be familiar about the current classifications, diagnosis and treatment modalities to best meet the needs of patients either self-reporting or diagnosed with this problem. Although, halitosis is one of the most common complaints with which patients come up to dental clinicians, thinking it can be detrimental to his/her self-image and confidence. Nevertheless, there have been a great number of studies conducted over the past three decades, it was somewhat surprising to find so few high quality randomized controlled trials comparing the effectiveness of some of these mouthrinses which effects on halitosis ${ }^{[30]}$. Clinical diverse among the studies was demonstrated by the inclusion of participants with low organoleptic and baseline volatile sulphur 
compounds in addition to those with significantly higher inpatients, those who had been referred to clinicians. However, the reference for evaluation of halitosis is organoleptic assessment, the comparatively accessible to use and convenience of the halimeter must be offset against its low sensitivity to significant malodourants other than hydrogen sulphide ${ }^{[31]}$. Mouthrinses with chlorhexidine have been shown to be successful in reducing antibacterial activity in supragingival plaque besides the bacterial load on the tongue and thus are seen as potentially effective agents in controlling halitosis ${ }^{32}$. The clinical effectiveness of a mouthrinse combining $0.05 \%$ chlorhexidine with cetylpyridinium chloride and zinc lactate was documented ${ }^{[32]}$. However, chlorhexidine also has some disadvantages predominantly with the increased tooth and tongue staining, bad taste and some reduction in taste sensation. The effectiveness of a two-phase oil-water mouthrinse containing $0.05 \%$ cetylpyridinium chloride was illustrated by productive reductions of organoleptically reviewed. Individuals depart in their perceptions of odor intensity, what each person believes objectionable varies considerably. In population where periodontal problems are more frequently untreated, halitosis may be more familiar, and equally acceptable. The reduction of bacterial levels in sites such as the tongue that might serve as reservoirs for odour producing bacteria is of paramount importance in controlling halitosis. Furthermore, it had have been enlightened that mouthrinses containing antibacterial agents such as chlorhexidine and cetylpyridinium chloride play an important role. The effectiveness of chlorinedioxide and zinc containing mouthrinses should not be underestimated. Therefore it appears to be a place in the management strategy of halitosis for formulations which include and combine some of these constituents may beneficial for the management of halitosis.

\section{Conclusion}

The role of dental practitioner with through knowledge of the general and oral variations in the diagnosis and treatment of halitosis is essential. Since of its personal nature, halitosis can cause social embarrassment, emotional and psychological distress leading to a lack of self-esteem, self-image, and selfconfidence. In conclusion, there is a need that dentists can help patients to handle the situation by giving counsel on oral hygiene, or can refer them for medical advice when a non-oral cause is deduced. As frontline health care workers, dentists were expected to stumble upon this kind of problem. Thus a holistic approach should be followed to improve the quality of life of our patients. Furthermore, there is growing interest and demand on halitosis, more research and studies are need to be done to explore, and to increase dentist knowledge of this condition.

References
[1] Bosy A, Kulkarni GV, Rosenberg M, McCulloch CA. Relationship of oral malodor to periodontitis: evidence of independence in discrete subpopulations. Journal of Periodontology 1994; 65(1): 37-46.

[2] Tonzetich J. Production and origin of oral malodor: a review of mechanisms and methods of analysis. Journal of Periodontology $1977 ; 48(1): 13-20$

[3] Sdsneyer L H, man W, Hmmfun L \& Gffmom R W. Rate of flow of human parotid, sublingual, and submaxillary secretions during sleep. J Dent Res. 1956 Feb; 35(1):109-14.

[4] Prfnz H. Offensive breath. Its causes and its prevention. Dent, Cosmos 1930; 72:70-7.

[5] Yaegaki K, Coil JM. Examination, classification, and treatment of halitosis; clinical perspectives. Journal of the Canadian Dental Association 2000; 66(5):257-61

[6] Miyazaki H, Sakao S, Katoh Y, Takehara T. Oral malodor in the general population of Japan. In: Rosenberg M editor(s). Bad breath: research perspectives. Tel Aviv: Ramot Publishing, 1995: 119-36.

[7] Meningaud JP, Bado F, Favre E, Bertrand JC, Guilbert F. Halitosis in 1999. Revue de Stomatologie et de Chirurgie MaxilloFaciale 1999; 100(5):240-4.

[8] Ayers KM, Colquhoun AN. Halitosis: causes, diagnosis, and treatment. The New Zealand Dental Journal 1998; 94(418):156-60.

[9] Delanghe G, Ghyselen J, van Steenberghe D, Feenstra L. Multidisciplinary breath-odour clinic. Lancet 1997; $350(9072): 187$.

[10] van Steenberghe D. Breath malodor. Current Opinion in Periodontology 1997; 4:137-43.

[11] Scully C, el-Maaytah M, Porter SR, Greenman J. Breath odor: etiopathogenesis, assessment and management. European Journal of Oral Sciences 1997; 105(4):287-93.

[12] Morita M, Wang HL. Association between oral malodor and adult periodontitis: a review. Journal of Clinical Periodontology 2001; 28 (9):813-9.

[13] Kleinberg I, Westbay G. Oral malodor. Critical Reviews in Oral Biology and Medicine 1990;1(4):247-59

[14] Kleinberg I, Codipilly M. In: Rosenberg M editor(s). The biological basis of oral malodor formation. Tel Aviv: Ramot Publishing, 1995:13-40.

[15] Ratcliff PA, Johnson PW. The relationship between oral malodor, gingivitis, and periodontitis. A review. Journal of Periodontology 1999; 70(5):485-9.

[16] Bosy A. Oral malodor: philosophical and practical aspects. J Can Dent Assoc 1997 March; 63(3): $196-201$.

[17] Replogle WH, Beebe DK. Halitosis. American Family Physician 1996; 53(4):1215-8, 1223.

[18] Stamou E, Kozlovsky A, Rosenberg M. Association between oral malodour and periodontal disease-related parameters in a population of 71 Israelis. Oral Diseases 2005; 11 Suppl 1:72-4

[19] Rosenberg M, McCullough CA. Measurements of oral malodor: current methods and future prospects. J Periodontol 1992; 63: 776-82.

[20] Nachnani S, Majerus G, Lenton P, Hodges J, Magallanes E.Effects of training on odor judges scoring intensity. Oral Dis 2005; 11: 40-4. 
[21] Greenman J, Duffield J, Spencer P, Rosenberg M, Corry D, Saad S et al.Study on the organleptic intensity scale for measuring oral malodor. J Dent Res 2004; 83: 81-5.

[22] Outhouse TL, Al-Alawi R, Fedorowicz Z, Keenan JV. Tongue scraping for treating halitosis. Cochrane Database of Systematic Reviews 2006 Apr 19; (2):CD005519.

[23] Richter JL. Diagnosis and treatment of halitosis. Compendium of Continuing Education in Dentistry 1996; 17(4):370-2, 374-6.

[24] Carvalho MD, Tabchoury CM, Cury JA, Toledo S, Nogueira-Filho GR. Impact of mouthrinses on morning bad breath in healthy subjects. Journal of Clinical Periodontology 2004; 31(2):85-90.

[25] Farrell S, Baker RA, Somogyi-Mann M, Witt JJ, Gerlach RW. Oral malodor reduction by a combination of chemotherapeutical and mechanical treatments. Clinical Oral Investigations 2006; 10(2): 157-63.

[26] Silwood CJ, Grootveld MC, Lynch E. A multifactorial investigation of the ability of oral health care products (OHCPs) to alleviate oral malodour. Journal of Clinical Periodontology 2001; 28(7):634-41.

[27] Tsunoda M, Sato H, Ohkushi T. The experimental study for the effect of sodium copper chlorophyllin in halitosis (author's tra nsl. Nippon Shishubyo Gakkai Kaishi Journal of the Japanese Association of Periodontology 1981; 23(3):490-8.

[28] Solis-Gaffar MC, Niles HP, Rainieri WC, Kestenbaum RC. Instrumental evaluation of mouth odor in a human clinical study. Journal of Dental Research 1975; 54(2):351-7.

[29] Pedrazzi V, Sato S, de Mattos Mda G, Lara EH, Panzeri H.Tonguecleaning methods: a comparative clinical trial employing a toothbrush and a tongue scraper. Journal of Periodontology 2004; 75 (7):1009-12.

[30] Fedorowicz Z, Aljufairi H, Nasser M, Outhouse TL, Pedrazzi V. Mouthrinses for the treatment of halitosis. Cochrane Database Syst Rev. 2008 8; (4):CD006701.

[31] Rassameemasmaung S, Sirikulsathean A, Amornchat C, Hirunrat K, Rojanapanthu P, Gritsanapan W. Effects of herbal mouthwash containing the pericarp extract of Garcinia mangostana L on halitosis, plaque and papillary bleeding index. Journal of the International Academy of Periodontology 2007; 9(1):19-25.

[32] Winkel EG, Roldan S, Van Winkelhoff AJ, Herrera D, Sanz M. Clinical effects of a new mouthrinse containing chlorhexidine, cetylpyridinium chloride and zinc-lactate on oral halitosis. A dualcenterdualcenter, double-blind placebo-controlled study. Journal of Clinical Periodontology 2003; 30(4):300-6.

Figure-1. Flowchart summarising the classification of halitosis

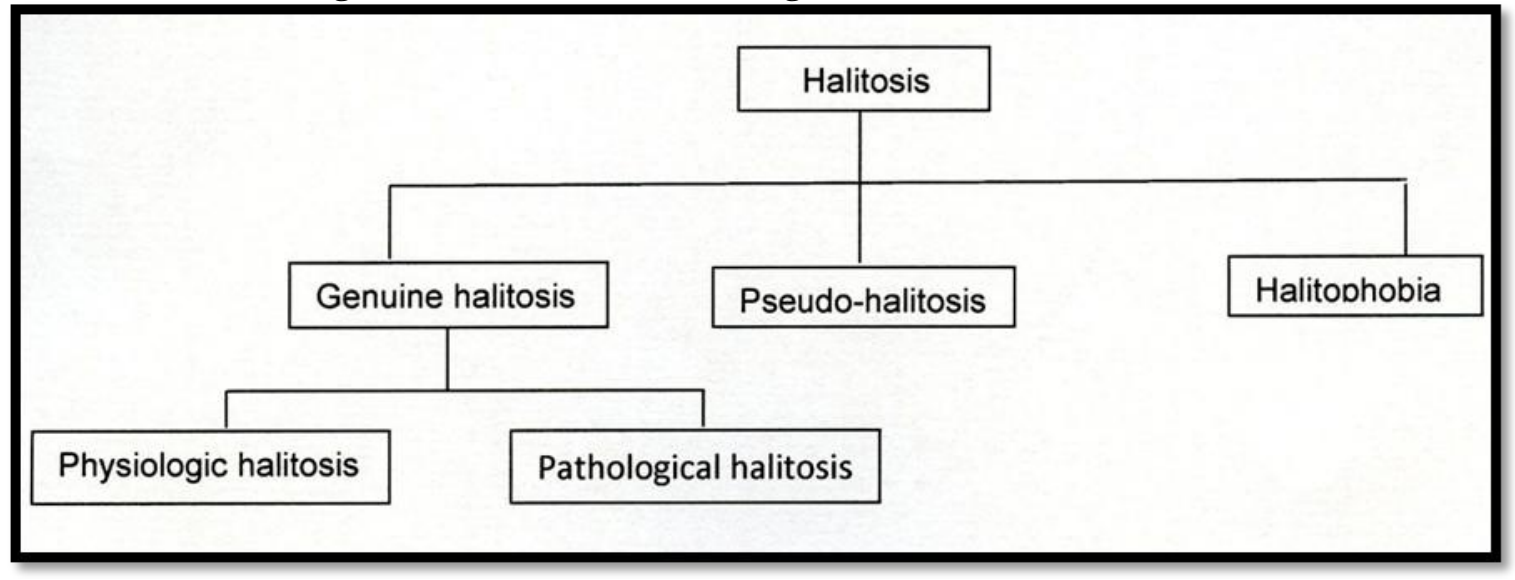

